\title{
Téoros
}

Revue de recherche en tourisme

\section{Analyse des cycles du tourisme américain au Québec}

\section{Jean Stafford}

Volume 9, numéro 3, novembre 1990

URI : https://id.erudit.org/iderudit/1079889ar

DOI : https://doi.org/10.7202/1079889ar

Aller au sommaire du numéro

Éditeur(s)

Université du Québec à Montréal

ISSN

0712-8657 (imprimé)

1923-2705 (numérique)

Découvrir la revue

Citer cet article

Stafford, J. (1990). Analyse des cycles du tourisme américain au Québec. Téoros, 9(3), 34-37. https://doi.org/10.7202/1079889ar d'utilisation que vous pouvez consulter en ligne.

https://apropos.erudit.org/fr/usagers/politique-dutilisation/ 


\section{Analyse des cycles du tourisme américain au Québec}

Très tôt, pour les visiteurs américains, le Québec a été une destination privilégiée; ils ont joué un grand rôle dans la création des premières stations touristiques. Ainsi, pendant longtemps, les touristes venant des États-Unis ont été les seuls étrangers que l'on pouvait rencontrer sur nos routes et dans nos villes pendant la saison touristique.

Aujourd'hui, cette situation a bien changée. De 1977 à 1988, la part des visiteurs américains dans l'ensemble des visitesprovince au Québec est passée de $88 \%$ a $82 \%$. Pour les mêmes périodes, la part des nuitées des Américains au Québec est descendue de $76 \%$ a $64 \%$. La place relativedes États-Unis dans le marché touristique québecois est en diminution depuis une dizaine d'années.

Il semble que, de plus en plus, le Québec et le Canada, du reste, ne sont que "1'arrièrecour touristique" des États-Unis; dans cette perspective, le Québec ne serait plus, au plan touristique, qu'une destination domestique pour les visiteurs américains.

\section{La notion de tendance}

Les hommes en général et les décideurs politiques en particulier ont une sainte horreur de la durée. Les uns et les autres vivent dans l'instantaneite du bulletin de nouvelles de la radio ou de la télévision et des manchettes des journaux quotidiens. L'événement est rarement révélateur, il est souvent un leurre; comme le souligne fortement Femand Braudel: " "... si on observe exclusivement dans 1'étroite actualité, l'attention ira vers ce qui bouge vite, brille à tort ou à raison, ou vient de changer ou fait du bruit, ou se révèle sans peine"(1). Dans l'ensemble, "1"événement bête et méchant" est rarement significatif; il apporte peu d'explicationet tend à substituer la réflexion sur un phénomène à une opinion sur celuici.

La notion de tendance est un excellent outil pourl'analyse du développement touristique car elle s'inscrit dans la moyenne et la longue durée. Dans ce sens: "Rechercher des tendances, c'est s'efforcer de déceler dans les chroniques certaines régularités, plus ou moins apparentes et simples, certains invariants liés à 1 'inertie des phénomènes économiques" ${ }^{\prime(2)}$. Les aspects récurrents de la vie économique sont aussi les supports de l'analyse du développement; ce qu'on appelle phénomène d'inertie, structures économiques, pesanteurs sociologiques ne sont que des parties de mouvements fondamentaux essentiels a la connaissance du changement. Ces eléments sont nécessaires; selon Bertrand de Jouvenel: "On peut affirmer que les prédictions - du moins les estimations de caractère scientifique - sont toujours engendrées au moyendupostulat que quelque chose restera constant. La constance de ce "quelque chose" est, à vrai dire, la "théorie" utilisée pour la prédiction" ${ }^{\text {?t(3). }}$

Plus concrètement, la tendance a moyen terme ou à long terme "... représente le mouvement de base de la série; elle ne peut donc comprendre des sommetset des creux successifs puisqu'elle schématise l'évolution essentielle, fondamentale de la grandeur mesurée. Elle représente le changement moyen par unité de temps de la variable analysée et est, à ce titre, un excellent indicateur de la croissance"t(4). C'est ce mouvement de base des séries étudiées qui nous permettra d'analyser le développement de l'industrie touristique au Québec.

Dans cet article, nous allons étudier les principales tendances du tourisme américain au Québec dans les dix dernières années. Nous allons aussi analyser les mouvements cycliques afin de visualiser les fluctuations longues propres au marché américain du tourisme au Québec.

\section{Les visites-province}

Dans le tableau 1 , nous avons les visitesprovince des touristes américains au Québec de 1977 à 1988. Le taux d'accroissement annuel moyen ${ }^{(5)}$, établi à partir des données observées, est de $2,3 \%$ par année. Le coefficient de variations ${ }^{(6)}$, entre 1977 et 1988 , est de $11 \%$, ce qui indique une relative stabilité. Le taux d'accroissement annuel moyen pour 1989-1993 sera de 5,3\%. 
TABLEAU 1

Prévision du nombre de visites-provinces(i) des touristes américains au Québec de 1977 à 1993

\begin{tabular}{|c|c|c|c|c|}
\hline \multirow{2}{*}{ Annés } & \multirow{2}{*}{$\begin{array}{l}\text { Visites- } \\
\text { province } \\
\text { observeses } \\
(000)\end{array}$} & \multirow{2}{*}{$\begin{array}{l}\text { Visites- } \\
\text { province } \\
\text { prévues }^{(0)} \\
(000)\end{array}$} & \multicolumn{2}{|c|}{ Intervalles deconfiance a $95 \%$} \\
\hline & & & $\begin{array}{c}\text { Inférieurs } \\
(000)\end{array}$ & $\begin{array}{l}\text { Superieurs } \\
(000)\end{array}$ \\
\hline 1977 & 2347000 & 2357828 & 2227210 & 2488445 \\
\hline 1978 & 2341000 & 2359128 & 2257342 & 2460915 \\
\hline 1979 & 2130000 & 2374591 & 2281728 & 2467453 \\
\hline 1980 & 2560000 & 2404215 & 2308070 & 2500360 \\
\hline 1981 & 2555000 & 2448000 & 2346212 & 2549788 \\
\hline 1982 & 2438000 & 2505947 & 2401749 & 2610144 \\
\hline 1983 & 2543000 & 2578055 & 2476267 & 2679843 \\
\hline 1984 & 2633000 & 2664325 & 2568179 & 2760470 \\
\hline 1985 & 2800000 & 2764756 & 2671893 & 2857618 \\
\hline 1986 & 2968000 & 2879348 & 2777562 & 2981134 \\
\hline 1987 & 3036000 & 3008102 & 2877485 & 3138720 \\
\hline 1988 & 3069000 & 3151018 & 2971921 & 3330114 \\
\hline 1989 & $=$ & 3308095 & 3064172 & 3552017 \\
\hline 1990 & $=$ & 3479333 & 3156580 & 3802086 \\
\hline 1991 & - & 3664733 & 3250399 & 4079066 \\
\hline 1992 & - & 3864294 & 3346283 & 4382305 \\
\hline 1993 & - & 4078016 & 3444588 & 4711445 \\
\hline
\end{tabular}

(1) Les viaites-prowince comptabilisent les touristes qui sont entrés au Canada via les frontieres des autres provinoes canadiennes mais dont une partie du voyege s'est faite au Ousbec (definition tiree de CLUZEAU, P. 1989, p. 32).

(2) Source: CLUZEAU, P., Le Québec touriatique, 1989.

(B) II s'agit d'une tendance parabolique.
TABLEAU 2

Prévision des nuitées des touristes américains au Québec de 1977 à 1993

\begin{tabular}{ccccc}
\hline Annés & $\begin{array}{c}\text { Nuitées } \\
\text { observées }\end{array}$ & $\begin{array}{c}\text { Nuités } \\
\text { prévues }\end{array}$ & \multicolumn{2}{c}{ Intervalles deconfiance à $95 \%$} \\
\cline { 4 - 5 } & $(000)$ & $(000)$ & $\begin{array}{c}\text { Inférieurs } \\
(000)\end{array}$ & $\begin{array}{c}\text { Supérieurs } \\
(000)\end{array}$ \\
\hline 1977 & 8106 & 8835 & 8402 & 9269 \\
1978 & 8625 & 8634 & 8296 & 8972 \\
1979 & 8388 & 8508 & 8200 & 8817 \\
1980 & 8704 & 8458 & 8139 & 8777 \\
1981 & 8687 & 8483 & 8146 & 8821 \\
1982 & 8289 & 8584 & 8238 & 8930 \\
1983 & 8646 & 8760 & 8422 & 9098 \\
1984 & 8952 & 9011 & 8693 & 9330 \\
1985 & 9520 & 9339 & 9031 & 9647 \\
1986 & 10091 & 9742 & 9404 & 10080 \\
1987 & 10322 & 10221 & 9787 & 10654 \\
1988 & 10435 & 10775 & 10181 & 11369 \\
1989 & - & 11405 & 10595 & 12215 \\
1990 & - & 12111 & 11039 & 13183 \\
1991 & - & 12893 & 11516 & 14269 \\
1992 & - & 13750 & 12029 & 15471 \\
1993 & - & 14684 & 12579 & 16789 \\
& & & & \\
\hline & & & &
\end{tabular}

(1) Sources: CLUZEAU, P., Le Québec touristique, 1989.

(2) Il s'agit d'une tendance de type parabolique.
Les baisses de touristes les plus importantes se situent en $1979 \mathrm{et}$, à un degré moindre en 1982. Il semble bien que la série a été peu touchée par le cycle macro-économique de 1982-1985. Les visites-province des touristes américains augmentent faiblement mais de façon graduelle; on devrait compter près de quatre millions de touristes en 1993.

\section{Les nuitées}

Les nuitées sont un indicateur important de l'industrie touristique. Dans le tableau 2, nous avons les prévisions des nuitées de 1977 à 1993. On peut noter une baisse de 237000 nuitées en 1979 et une autre de 398 000 nuitées en 1982. La croissance annuelle des nuitées est de $2,3 \%$ entre 1977 et 1988 .

Au niveau des prévisions, on peut s'attendre à une progression de $6,5 \%$ des nuitées, par année, de 1989 à 1993. Le coefficient de variationest de $8,8 \%$. Selon cette tendance, il y aurait donc près de 14 millions de nuitées en 1993.

\section{Les recettes du tourisme américain au Québec}

Dans le tableau 3, nous avons l'évolution des recettes (en dollars constants de 1981) du tourisme américain au Québec. Pour les valeurs observées, de 1977 à 1988, l'augmentation des recettes est, en moyenne par année, de $4 \%$. Le coefficient de variation est de $38,6 \%$.

De 1989 à 1993, le modàle prévoit une croissance de 3,3\% par annee des recettes touristiques avec les États-Unis. Dès 1990, ces revenus vont atteindre le demi-milliard de dollars (dollars constants de 1981). On remarque aussi une baisse assez importante des recettes en 1982; depuis 1983, l'evolution est graduelle.

\section{Le deficit touristique du Québec avec les États-Unis}

Le déficit touristique du Québec avec les États-Unis a connu (en dollars constants de 1981) une baisse annuelle de $-2,8 \%$ entre 1977 et 1988 (voir le tableau 4). Le coefficient de variation est de $30,8 \%$.
Le modelle prévisionnel suppose une décroissance annuelle de $-1,95 \%$, par année, entre 1989 et 1993 . Si cette tendance se réalise, à moyen terme, le déficit touristique avec les États-Unis pourrait être contenu dans des limites raisonnables.

\section{Le mouvement cyclique du tourisme américain au Québec}

Les hausses et les baisses des arrivées de touristes américains ou dans les dépenses de ces visiteurs laissent supposer l'existence de cycles. Ces cycles sont des fluctuations à moyen terme ou à long terme autour de la tendance principale ${ }^{(\pi)}$.

Nous avons construit des indices cyclique ${ }^{\left({ }^{(3)}\right)}$, ils apparaissent dans le tableau 5. Pour tous les indicateurs, il $y$ a de faibles écarts par rapport à 100 (et à l'exception du déficit touristique). La moyenne des variations est de $4,2 \%$ pour les visites-province et de $2,5 \%$ pour les nuitées. Pour les recettes, les ecarts varient, en moyenne, de $3,3 \%$ et de $23 \%$ pour le déficit. Ces faibles fluctuations apparaissent nettement dans le graphique 1 . 


\section{TABLEAU 3}

Prévision des recettes des touristes américains au Québec, en dollars constants de 1981, de 1977 à 1993

\begin{tabular}{|c|c|c|c|c|}
\hline \multirow[t]{2}{*}{ Années } & \multirow{2}{*}{$\begin{array}{l}\text { Recettes } \\
\text { observées }\end{array}$} & \multirow{2}{*}{$\begin{array}{l}\text { Recettes } \\
\text { prévues }^{(2)} \\
(000000)\end{array}$} & \multicolumn{2}{|c|}{ Intervalles de confiance a $95 \%$} \\
\hline & & & $\begin{array}{l}\text { Inferieurs } \\
(000000)\end{array}$ & $\begin{array}{l}\text { Supérieurs } \\
(000000)\end{array}$ \\
\hline 1977 & 275 & 278 & 258 & 298 \\
\hline 1978 & 303 & 295 & 278 & 313 \\
\hline 1979 & 296 & 312 & 297 & 327 \\
\hline 1980 & 359 & 330 & 316 & 342 \\
\hline 1982 & 358 & 346 & 335 & 358 \\
\hline 1982 & 340 & 363 & 353 & 374 \\
\hline 1983 & 359 & 381 & 370 & 391 \\
\hline 1984 & 397 & 398 & 386 & 409 \\
\hline 1985 & 421 & 415 & 402 & 428 \\
\hline 1986 & 447 & 432 & 417 & 447 \\
\hline 1987 & 455 & 449 & 432 & 467 \\
\hline 1988 & 458 & 466 & 446 & 486 \\
\hline 1989 & - & 483 & 461 & 506 \\
\hline 1990 & - & 501 & 475 & 526 \\
\hline 1991 & - & 518 & 489 & 546 \\
\hline 1992 & - & 535 & 503 & 566 \\
\hline 1993 & - & 552 & 517 & 586 \\
\hline \multicolumn{5}{|c|}{$\begin{array}{l}\text { Source: CLUZEAU, Pu, Le Québec touristíque, 1989. Les différences entre } \\
\text { ces chiffres et ceux de Patrick Cluzeau s'expliquent par le fait que le présent } \\
\text { tableau est construita partir des recettes en dollars constants de } 1981 \text { et non } \\
\text { en dollars courants } \\
\text { II s'agit d'une tendance linéaire. }\end{array}$} \\
\hline
\end{tabular}

TABLEAU 4

Prévision du déficit touristique du Québec avec les ÉtatsUnis, en dollars constants de 1981, de 1977 à 1993

\begin{tabular}{|c|c|c|c|c|}
\hline \multirow{2}{*}{ Années } & \multirow{2}{*}{$\begin{array}{c}\text { Déficits } \\
\text { observés }{ }^{(1)} \\
(000000)\end{array}$} & \multirow{2}{*}{$\begin{array}{l}\text { Déficits } \\
\text { prevus }^{(2)} \\
(000000)\end{array}$} & \multicolumn{2}{|c|}{ Intervalles de confiance a 9} \\
\hline & & & $\begin{array}{l}\text { Inférieurs } \\
(000000)\end{array}$ & $\begin{array}{l}\text { Supérieurs } \\
(000000)\end{array}$ \\
\hline 1977 & 304 & 256 & 213 & 303 \\
\hline 1978 & 238 & 252 & 212 & 293 \\
\hline 1979 & 185 & 164 & 115 & 212 \\
\hline 1980 & 212 & 242 & 208 & 276 \\
\hline 1981 & 214 & 237 & 205 & 270 \\
\hline 1982 & 159 & 149 & 113 & 184 \\
\hline 1983 & 232 & 228 & 192 & 263 \\
\hline 1984 & 110 & 139 & 106 & 172 \\
\hline 1985 & 84 & 134 & 100 & 168 \\
\hline 1986 & 121 & 129 & 93 & 165 \\
\hline 1987 & 180 & 124 & 84 & 165 \\
\hline 1988 & 221 & 203 & 143 & 263 \\
\hline 1989 & - & 198 & 132 & 264 \\
\hline 1990 & - & 198 & 132 & 264 \\
\hline 1991 & - & 193 & 120 & 265 \\
\hline 1992 & - & 188 & 109 & 267 \\
\hline 1993 & - & 183 & 97 & 269 \\
\hline
\end{tabular}

(1) Source: CLUZEAU, P., Le Oudbec touristique, 1989. Patrick Cluzeau caleule les déficits en dollars courants tandis que le présent tableau est d́tabli sur la base des dollara conatanta de 1981

(2) II s'agit d'une tendance linéaire couplée à un cycle conjoncturel.
On remarque aussi, dans le tableau 5 , que pour les visites-province, les périodes de baisse se situent en 1979 et ensuite en 1982 , en 1983 et en 1984; il y a donc 8 années de hausse et quatre années de baisse. Pour les nuitées, les années difficiles se trouvent en 1977,1978 et 1979 ; ces périodes se reflètent en 1982, 1983 et 1984 et ensuite en 1988. On retrouve dans cette série 7 années de récession et 5 années de relative prospérité.

En ce qui conceme les recettes, les années fortes sont 1977 et 1978,1980 et 1981 et par la suite de 1984 à 1987 ; les années faibles sont en 1979 , en 1982 et 1983 , et enfin en 1988. On compte ici encore 8 années de hausse et 4 années de baisse. Le comportement du déficit est assez différent; on remarque tout d'abord de très fortes fluctuations du deficit pendant ces 12 années. Les périodes de baisse sont en 1979 , en 1982 et de 1984 à 1986; les périodes de hausse sont en 1977 et 1978 , en 1980 et 1981, en 1983 et enfin en 1987 et 1988 . II y a donc 7 années de hausse et 4 années de baisse.

En utilisant les mêmes indicateurs cycliques pour les touristes étrangers (a l'exception des touristes des États-Unis), on obtient des résultats très différents ${ }^{(9)}$. Les fluctuations des séries étrangères (sans tenir compte des États-Unis) sont très accentuées; ce sont des variations assez fortes sur des périodes de temps assez longues. La vigueur de ces oscillations contraste avec l'évolution des cycles du tourisme américain au Québec.

De plus, dans ces séries statistiques des touristes étrangers, les périodes de hausse et les périodes de baisse sont très souvent inversées par rapport aux cycles du tourisme américain. Par exemple, les années de baisse du tourisme américain correspondent à des années de hausse du tourisme étranger; ce phénomène est observable pour beaucoup d'indicateurs cycliques. Il faut garder à l'esprit que les mouvements alternatifs (de hausse ou de baisse) sont toujours plus faibles pour le tourisme américain au Québec.

\section{Le Québec: un marché domestique pour les États-Unis}

Il semble bien que le secteur du tourisme (comme dans beaucoup d'autres secteurs de l'économie québécoise) soit un marché domestique pour les touristes américains.
Cette tendance s'exprime dans la faiblesse relative des taux $d$ 'accroissement annuel moyen et dans les très faibles variations cycliques. Il s'agit d'un marché sans. surprise et sclérosé.

Cette situation résulte d'une part de la forte influence de la culture américaine sur le Québec et le Canada. Cette influence, qui s'exerce par les produits de consommation courants et par les médias, entraîne une forte banalisation, pour les Americains, des produits touristiques québécois.

Mềme si on se sent en sécurité, il n'est pas toujours intéressant de passer ses vacances annuelles dans l'arrière-cour de notre résidence. On a souvent l'impression que c'est de cette façon que le Québec est perçu par les touristes américains. Cette faible spécification du produit (que cette faiblesse soit réelle ou imaginaire) amène une certaine stagnation de nos relations avec notre puissant voisin.

Il faut dire, pour terminer, que cette perception n'est pas propre aux Américains. Beaucoup de Québécois se sentent chez eux dans les États de la NouvelleAngleterre, en Floride ou ailleurs aux ÉtatsUnis. Les États-Unis (et le Québec pour les 


\section{TABLEAU 5}

Indices cycliques du tourísme américain au Québec, de 1977 à 1988

\begin{tabular}{|lcrrr|}
\hline Années & $\begin{array}{c}\text { Visites- } \\
\text { province }\end{array}$ & Nuitées & Recettes & Déficit \\
\hline 1977 & 103 & 92 & 108 & 122 \\
1978 & 100 & 99 & 103 & 101 \\
1979 & 88 & 98 & 91 & 84 \\
1980 & 103 & 103 & 104 & 103 \\
1981 & 100 & 102 & 102 & 113 \\
1982 & 95 & 96 & 95 & 91 \\
1983 & 95 & 99 & 95 & 137 \\
1984 & 96 & 99 & 100 & 66 \\
1985 & 100 & 102 & 100 & 51 \\
1986 & 103 & 103 & 102 & 75 \\
1987 & 104 & 100 & 101 & 114 \\
1988 & 102 & 97 & 99 & 143 \\
\hline
\end{tabular}

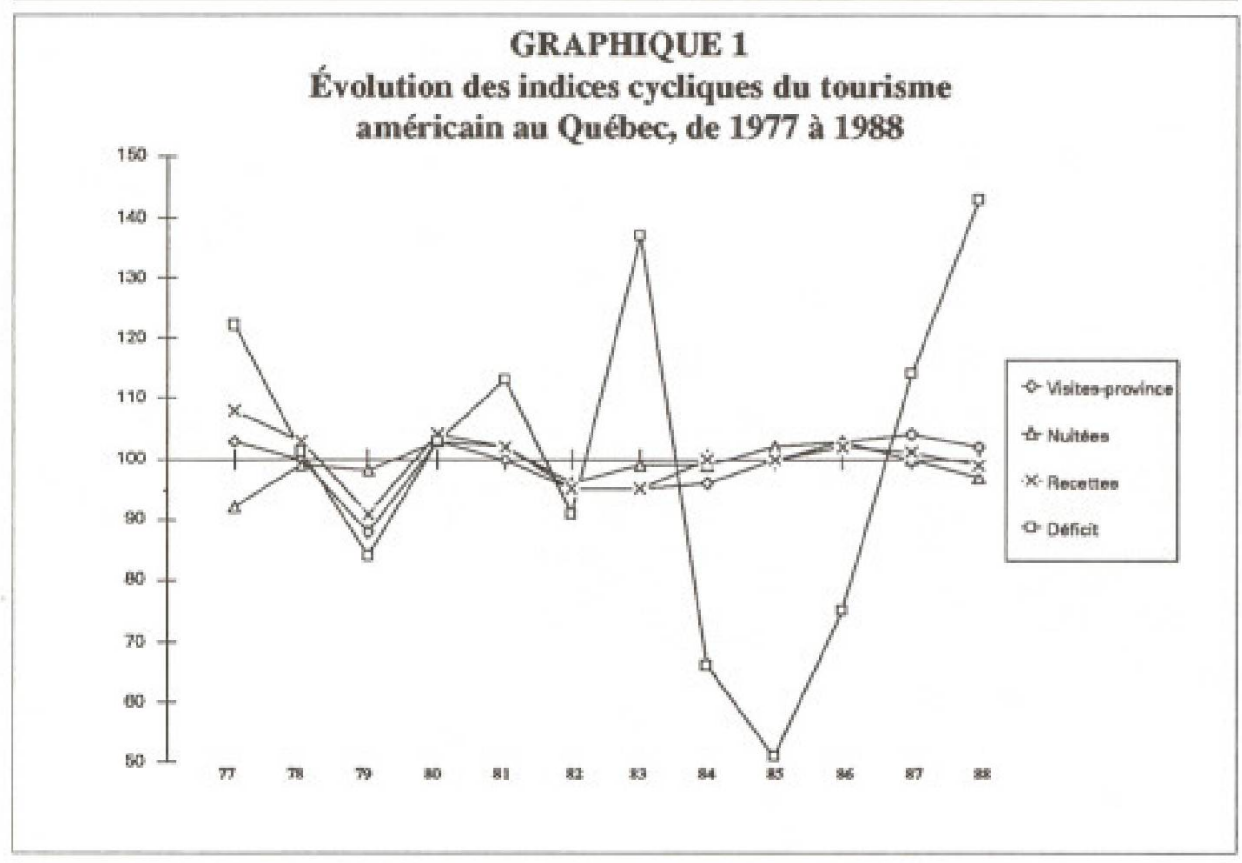

Américains) ressemblent à ce voisin de palier que l'on voit tous les jourset que l'on ne connaît pas tout en ayant l'impression de le connaître. Le grand probleme est de restaurer la distance culturelle et physique pour redonner au Québec du mystère et du charme. $f$

\section{NOTES}

(1) Braudel, F., Ecrits sur l'hlsteire, Flammarion, 1969 , $\mathrm{p}, 59$; voli aussi VOVELLE, M.t L'histoire et a longue durde, dans La nouvelle histoirs (2ous La direction de J. Le-Gotf), Las Encyelopédies du Savoir Modeme, RETZ, 1978.

(2) NAHON, G., SALOMON, M., L'slaboration des provisions do march6, Dunod, 1977, p. 21.

(3) Jowvenel, B., L'art de la conjecture, Éditione du Riocher, 1964, p. 232.

(4) GUERIN, G., Des stries chronologiques au systeme statistlque eansdien, Gadtan Morin Ed., 1963 , p. 21.

(5) Pour plus de précision, nous utilisons, dana le calcul du taux d'accroisaement annuel moyen la moyenne gémétrique. La fermule utilisesest: $\operatorname{TAMM}=\sqrt[n]{\mathrm{n}^{+1} / \mathrm{m}^{0}}$
(6) Pour comparer les fluctuationa dens los différentes séries chronologiques, nous utilisons le coefficient de variation:-colu-ci consiste à diviser l'écantype de la atrie par la moyenne de la série; le ooefficient de variation s'bcrit:

$C V=\frac{g}{x}$

i7) Voir at ce sujet: GRANELLE, J, Fluctuations économiques et conjoneture, Masson, 1987: ot JARRETT, J, Business Forecasting Mothods, Basil Blackwell Ine., 1987.

(6) La construction des indices cycliques s"est faite â partir de différentes méthodes de lissage: -pardes tendances mathdmatiques de type linkaire ou parabolique (par annee):

- par diversea moyennas mobiles feurtout des moyernes mobiles doubles).

Dans tous les cas, ta formule utilisde ost:

$\operatorname{ICY}=(\underset{Y \mathrm{pr}}{\mathrm{Yi}} \times 100)$

où:

ICY = indice cyclique:

yi = valeurs observés:

Ypr = valeurs próvues avec une méthode quelconque.

(9) Stafford, J., Crises et doveloppement de ITnduatrie tourlatique au Qubbec a paraitre â Fautomne 1990. 